\title{
RESEARCH ON ESTIMATION CROP PLANTING AREA BY INTEGRATING THE OPTICAL AND MICROWAVE REMOTE SENSING DATA
}

\author{
Y. Jia ${ }^{*}$, F. Yu \\ Chinese Academy of Surveying and Mapping, Beijing 100830, P. R. China - (jiayi, yufan)@casm.ac.cn
}

Topics: Multi-source classification and information extraction

KEY WORDS: Optical and microwave remote sensing, Normalization, Bayesian network, Classification, Crop planting area

\begin{abstract}
:
Considering the problem in monitoring agricultural condition in the semi-arid areas of Northwest of China, we propose a new method for estimation of crop planting area, using the single phase optical and microwave remote sensing data collaboratively, which have demonstrated their respective advantages in the extraction of surface features. In the model, the ASAR backscatter coefficient is normalized by the incident angle at first, then the classifier based on Bayesian network is developed, and the $\mathrm{VV}, \mathrm{VH}$ polarization of ASAR and all the 7 TM bands are taken as the input of the classifier to get the class labels of each pixel of the images. Moreover the crop planting areas can be extracted by the classification results. At last, the model is validated for the necessities of normalization by the incident angle and integration of TM and ASAR respectively. It results that the estimation accuracy of crop planting area of corn and other crops garden are $98.47 \%$ and $78.25 \%$ respectively using the proposed method, with an improvement of estimation accuracy of about $3.28 \%$ and $4.18 \%$ relative to single TM classification. These illustrate that synthesis of optical and microwave remote sensing data is efficient and potential in estimation crop planting area.
\end{abstract}

\section{INTRODUCTION}

Agriculture is the basis of China's national economy, agricultural information monitoring is important for agricultural production, it is the basis for national socioeconomic information of the people's livelihood [1]. Estimation of Agricultural crop area is one of the key technologies to monitor the agricultural situation, the traditional methods is based on a sample survey which is human-based, point-based data. It spends a lot of money and has the low efficiency. The survey result is easily influenced by human factors. Based on the principle of electromagnetic waves emitted or reflected from an object on earth observation technology, Remote sensing technology can provide long-lasting surface information frequently, with macro, dynamic and accurate monitoring of the changes of the surface environment. so the use of remote sensing to establish an objective, dynamic, real- time, low-cost crop area estimation method is very significant.

Currently, the theory of estimating crop acreage by remote sensing data is mainly based on the spectral theory of green plants, using multi-temporal Landsat remote sensing data (Landsat TM) and very high resolution remote sensing data Meteorology (NOAA / AVHRR), methods there are greenness - phase - area mode, visual interpretation calculation method, the combination of remote sensing and statistical methods, combination of geographic information systems and remote sensing methods [2-4]. The United States is the world's first country to estimating crop area by remote sensing in 1974 through the "large-scale crop yield estimation experiment" (LACIE), Landsat MSS was used on wheat acreage estimation with precision up to $90 \%$ [5] Since 1988, the European Union carried out monitoring the EU's arable land, crop acreage and production using remote sensing technology through the MARS program, 
and the monitoring results were used for the verification of the declaration of agricultural subsidies and the reform of the Common Agricultural Policy [6]. China also actively carried out the work of Agricultural Remote Sensing. In 20 mid-1980s, the National Meteorological Administration estimated the production of northern winter wheat by NOAA / AVHRR satellite remote sensing yield over 11 provinces, the standard methods of estimating crop acreage by remote sensing is established [7]. Fang Hongliang [8] used supervised classification and unsupervised classification to extract for rice planting area by TM satellite data, the accuracy is more than $84 \%$. Wu Bing-Fang [4] built 'Chinese Agricultural Information of Remote Sensing Systems' in 1998, the system used remote cluster sampling and ground surveys of combining methods to estimate crop acreage, the crop acreage for bulk extraction accuracy was over $95 \%$ in nationwide.

The above-mentioned method requires more temporal optical remote sensing data, and optical data is easily influenced by weather, atmospheric conditions in the study area are higher, we are often difficult to obtain a high-quality crop growth phase continuous data. The radar data can pass through clouds and fog, with the alltime, all-weather characteristics, information from different perspectives reflects surface characteristics. If we combine the optical and radar data together to extract surface information, it is great significant for improving the extraction accuracy of crop area.

Based on this consideration, we propose a singlephase optical radar remote sensing of crop acreage synergistic extraction method. At first, the incidence angle of ASAR data is normalized, the backscattering coefficient were corrected in the same incident standards; and then a Bayesian networks is build, training areas were selected. ASAR dual polarization and TM7 bands were the input data as classification, based on the classification results to extract crop acreage. With the support of the measured data, we verified the results.

\section{Normalization of the ASAR backscattering coefficient by the incident angle}

The incident angle of SAR influences the signal intensity very much. Backscattering coefficient of the same object is different at vary incident angles, the discrepancy in vary situation (such as bare ground or vegetation-covered areas) shows unlikeness, which is not conducive to the land surface classification. So in order to make the surface objects under the uniformity incident angle standards and compare them, we normalized the ASAR backscattering coefficient by its incident angle.

2.1 Extrapolation of the optimal incident angle $\theta_{j}$ for Normalization

If the range of incident angles are $\left(\theta_{1}, \theta_{2}\right)$ in a SAR image, we need to find a $\theta_{j}$,which makes the changes of backscattering coefficient of each pixels after normalization minimized.

By the Taylor formula, it is possible to extrapolate the SAR backscattering coefficient to the optimal angle $\theta_{j}$, As $\theta \in\left(\theta_{1}, \theta_{2}\right)$, the error $E r$ for choosing $\theta_{j}$ as the optimal angle can be written as:

$E_{r}=\int_{\theta}^{\theta_{2}}\left(\sigma(\theta)-\sigma\left(\theta_{j}\right)\right)^{2} d \theta=\int_{\theta}^{\theta_{2}}\left(\sigma^{\prime}\left(\theta_{j}\right)\left(\theta-\theta_{j}\right)+\frac{1}{2} \sigma^{\prime \prime}\left(\theta_{j}\right)\left(\theta-\theta_{j}\right)^{2}+\xi\right)^{2} d \theta$

Where $\xi$ is the high-order terms of Taylor formula, when $\frac{d E_{r}}{d \theta_{j}}=0, E r$ is the minimum, the optimum angle can be found, then

$$
\left(\sigma_{j}\left(\theta_{2}-\theta_{j}\right)+\frac{1}{2} \sigma^{\prime}\left(\theta_{j}\right)\left(\theta_{2}-\theta_{j}\right)^{2}+\xi\right)^{2}=\left(\sigma^{\prime}\left(\theta_{j}\right)\left(\theta_{j}-\theta_{1}\right)+\frac{1}{2} \sigma^{\prime}\left(\theta_{j}\right)\left(\theta_{j}-\theta\right)^{2}+\xi\right)^{2}
$$

Because the common factor $\theta_{2}-\theta_{j}$ and $\theta_{j}-\theta_{1}$ can be extracted from $\xi_{2}$ and $\xi_{1}$ respectively, when $\theta_{2}-\theta_{j}=\theta_{j}-\theta_{1}$, the formula (2) can only be solved, and the solution is $\theta_{j}$ $=\left(\theta_{1}+\theta_{2}\right) / 2$

Previous researches [9] show that the land vegetation coverage affects the SAR backscattering coefficient slightly when NDVI $<0.45$ in $\mathrm{C}$ bands, and the backscattering is mainly composed by direct scattering 
from bare ground. On the contrary, when NDVI $>0.45$, the direct scattering of bare ground is less, and the vegetation scattering plays a leading role. Therefore, it is necessary to normalize the SAR backscattering coefficient in bare ground and vegetation-covered areas respectively.

\section{2 Normalization in bare ground or sparse vegetation} areas

From the previous researches [10], we know that the simulations of AIEM (Advanced Integral Equations Model) are identical with the field measurements, so AIEM is used to estimate the backscattering coefficient in different surface roughness, soil moisture content, and incident angles in bare soil or sparse vegetation. The single backscattering coefficient $\sigma_{p q}$ is given by:

$$
\sigma_{p q}=\frac{k_{1}^{2}}{2} \exp \left(-s^{2}\left(k_{z}^{2}+k_{s z}^{2}\right)\right) \sum_{n=1}^{\infty} \frac{s^{2 n}}{n !}\left|I_{p q}\right|^{2} W^{n}\left(k_{s x}-k_{x}, k_{s y}-k_{y}\right)
$$

Where $k_{1}$ is the wave number in medium $1 ; s$ is RMS height; $W^{n}\left(k_{s x}-k_{x}, k_{s y}-k_{y}\right)$ is the roughness spectrum of the surface related to the $n$th power of the surface correlation function by the Fourier transforms. $k_{z}=k \cos \theta_{i} ; k_{s z}=$ $k \cos \theta_{s} ; k_{x}=k \sin \theta_{i} \cos \varphi ; k_{s x}=k \sin \theta_{s} \cos \varphi_{s} ; k_{y}=k \sin \theta_{i} \sin \varphi ;$ $k_{s y}=k \sin \theta_{s} \sin \varphi_{s} ; \varphi$ is the incident azimuth angle; $\theta_{s}$ and $\varphi_{s}$ are the scattering angle and scattering azimuth angle, respectively; $I_{p q}{ }^{n}$ is a function of $\theta_{i}, \varphi, \theta_{s}, \varphi_{s}, \varepsilon_{s}, s$ and the Fresnel reflectivity.

The input parameters of AIEM are $\varepsilon_{s}$ which is calculated by the surface soil moisture $m_{v}$ and soil texture [11], $\theta_{i}, s$, correlation length $(l)$ and the surface correlation function $\rho(x)$, which is determined by analyzing the surface profile of each site.

In this paper, the simulated inputs of AIEM are set: $\mathrm{C}$ band, VV polarization, and the other input parameters are as follows (Table 1):

Table 1 The input parameters of simulation by AIEM

\begin{tabular}{cccc}
\hline Inputs & Min $\quad$ Max Step Unit
\end{tabular}

\begin{tabular}{ccccc}
\hline Soil Moisture $m_{v}$ & $5.0 \%$ & $40.0 \%$ & $3.0 \%$ & $\mathrm{~g} / \mathrm{cm}^{3}$ \\
RMS height $s$ & 0.1 & 4.0 & 0.25 & $\mathrm{~cm}$ \\
Correlation length $l$ & 5.0 & 25.0 & 2.5 & $\mathrm{~cm}$ \\
$\quad$ Incident & 15 & 45 & 3 & $\circ$ \\
angle $\theta_{i}$ & & & & \\
\hline
\end{tabular}

By analysis of the simulation results, we find when the roughness is $\operatorname{small}((s<2 \mathrm{~cm})$, there are obvious correlation between the backscattering coefficients $\left(\sigma\left(\theta_{m}\right)\right.$ and $\left.\sigma\left(\theta_{n}\right)\right)$ in different incident angles $\left(\theta_{m}\right.$ and $\left.\theta_{n}\right)$, There is a good liner relationship between $\sigma\left(\theta_{m}\right)$ and $\sigma\left(\theta_{n}\right)$, but the relationship is unreliable when the $\left(\left|\theta_{m}-\theta_{n}\right|\right)$ rises, if we try to directly establish the linear relationship between $\sigma\left(\theta_{i}\right)\left(\theta_{i} \in\left[15^{\circ}, 45^{\circ}\right]\right)$ and $\sigma\left(\theta_{j}\right)$ (backscattering coefficients at optimum angle $\theta_{j}$ ), it may cause large errors, which shows in Fig.1:

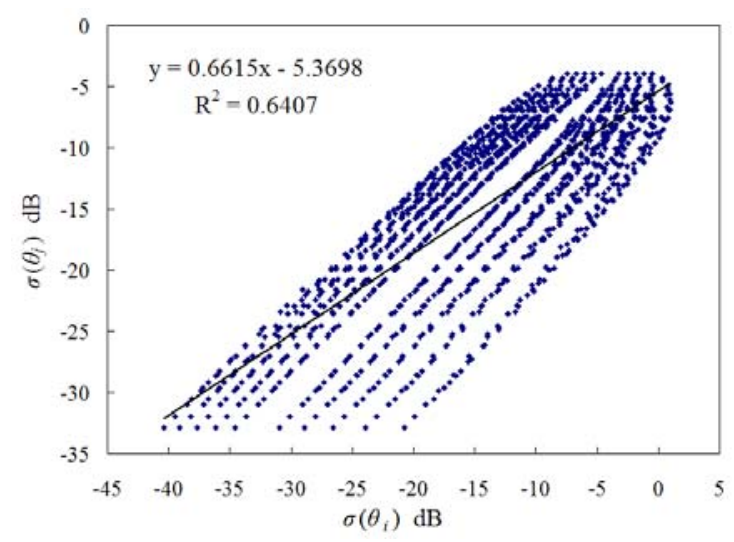

Figure. 1 Linear simulation between $\sigma\left(\theta_{j}\right)$ and $\sigma\left(\theta_{i}\right), \theta_{i} \in\left[15^{\circ}\right.$, $\left.\left.45^{\circ}\right]\right)$, step $3^{\circ}$

Then, according to the influence of the incident angles, the cosine and sine of incident angles are adopted to develop the empirical relationship between $\sigma\left(\theta_{j}\right)$ and $\sigma\left(\theta_{i}\right)$, in this paper, $\theta_{i} \in\left[15^{\circ}, 45^{\circ}\right]$, the empirical formula can be written as:

$$
\sigma\left(\theta_{j}\right)=a \frac{\cos \theta_{i}}{\cos \theta_{j}} \sigma\left(\theta_{i}\right)+b \ln \left(\frac{\sin \theta_{i}}{\sin \theta_{j}}\right)+c
$$

Where $a, b$ and $c$ are empirical coefficients, they changes when the rang of incident angles changes. In this research, $\theta_{j}=\left(15^{\circ}+45^{\circ}\right) / 2=30^{\circ}$. We can get the values of the empirical coefficients: $a=1.045, b=11.82$ and $c=$ 
0.004. Fig. 2 is the comparison of normalized backscattering coefficient $\sigma\left(\theta_{i}, 30^{\circ}\right)$ and $\sigma\left(30^{\circ}\right)$ simulated by AIEM:

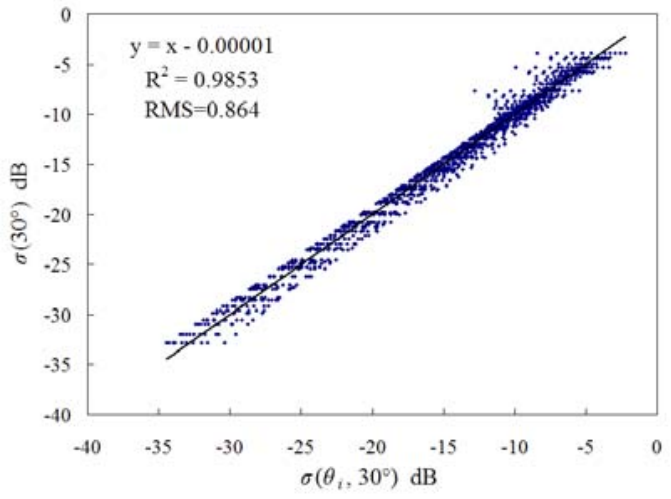

Figure. 2 Comparison of normalized backscattering coefficient $\sigma\left(\theta_{i}\right.$, $\left.30^{\circ}\right)$ and $\sigma\left(30^{\circ}\right)$ simulated by AIEM

In Fig. 2, the $\mathrm{R}^{2}$ is 0.9853 , and $\mathrm{RMS}$ is $0.864 \mathrm{~dB}$, it reveals the normalization formula (4) can gain a good precision at a large range of incident angles $\left(\theta_{i} €\left[15^{\circ}\right.\right.$, $\left.\left.45^{\circ}\right]\right)$.

\section{3 Normalization in vegetation-covered areas}

The water-cloud model [13] is introduced to simulated the backscattering in vegetation-covered areas. In the water-cloud model, The vegetation is represented as a homogeneous horizontal cloud of identical water spheres, the backscattering coefficient of whole canopy $\sigma_{c a n}$ is presented as the sum of the contribution of the vegetation $\sigma_{v e g}$ and the contribution of the underlying soil $\sigma_{\text {soil }}$, which is attenuated by the vegetation layer. Multiple scattering between canopy and soil can be neglected. For a given incidence angle $\theta_{i}$, the backscattering coefficient is represented in water-cloud models by the general form:

$$
\begin{gathered}
\sigma_{\text {can }}\left(\theta_{i}\right)=\sigma_{v e g}\left(\theta_{i}\right)+\gamma^{2}\left(\theta_{i}\right) \sigma_{\text {soil }}\left(\theta_{i}\right) \\
\gamma^{2}\left(\theta_{i}\right)=\exp \left(-2 B m_{v} / \cos \theta_{i}\right) \\
\sigma_{v e g}\left(\theta_{i}\right)=A \cos \theta_{i}\left(1-\gamma^{2}\left(\theta_{i}\right)\right)
\end{gathered}
$$

Where $\gamma^{2}\left(\theta_{i}\right)$ is the two-way transmissivity of the vegetation canopy, A and B are empirical coefficient determined by the vegetation type and the frequency of incident electromagnetic waves.

In vegetation-covered areas, NDVI $>0.45$, the total backscattering mainly consist of the scattering contribution of the vegetation layer. If the underlying ground scattering $\sigma_{\text {soil }}\left(\theta_{i}\right)$ is neglected, the $\gamma^{2}\left(\theta_{i}\right)$ tends to 0 , so $\sigma_{c a n}\left(\theta_{i}\right)=\sigma_{v e g}\left(\theta_{i}\right)=A \cos \theta_{i}$. Then the normalization formula in vegetation-covered areas can be expressed as:

$$
\sigma\left(\theta_{j}\right)=\frac{\cos \theta_{j}}{\cos \theta_{i}} \sigma\left(\theta_{i}\right)
$$

Similarly, the formulas (4) and (8) also can be used at $\mathrm{HH}$ or cross polarization, so we can obtain the normalization formulas of SAR backscattering coefficient at all polarization state.

\section{4 Classification method}

This paper presents a method based on integration of both active and passive remote sensing data for monitoring crop planting area. The experiment is carried out in Heihe river basin, a semi-arid area in the northwest of china. This method takes good use of the advantages from both optical and microwave remote sensing. The flow chart of the soil moisture extraction scheme is given in Figure 3.

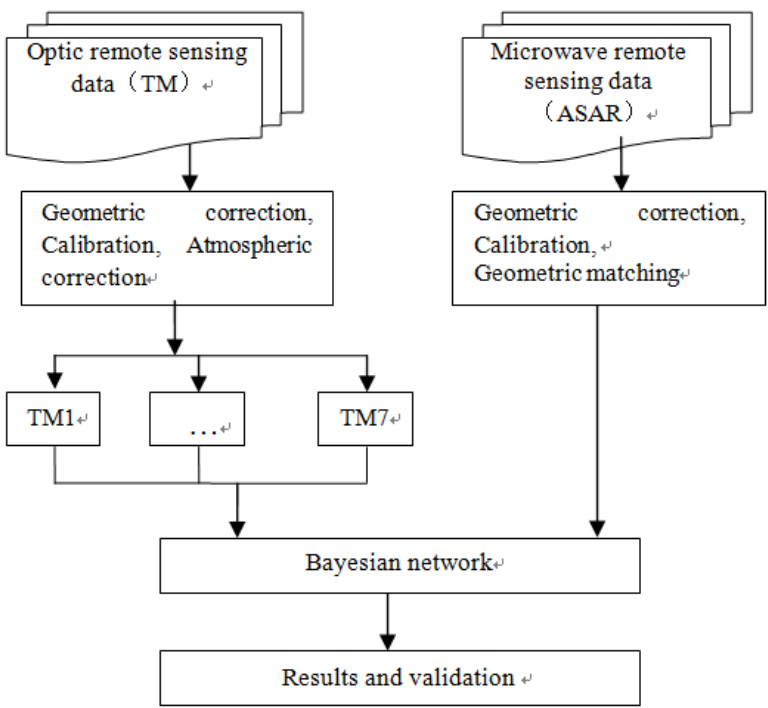

Figure 3. The flow chart of our method

\section{Study area and database}

The measures reported in this study are conducted during the Watershed Airborne Telemetry Experiment. The study area locates in Grass Station of Lanzhou University in Zhang Ye district, Gansu province; it is a part of the low plain of Hexi Corridor. Its geographical coordinates are $39.25043^{\circ} \mathrm{N}, 100.005871^{\circ} \mathrm{E}$, and the 
altitude is 1385 meters. Land use mainly consists of country, bare salinized land, irrigative agricultural fields, small piece of desert and proluvium fan. The field experiment was conducted from June to July in 2008, at which time the main crops were corn, while wheat, clove, barley and other crops can also be found.

Satellites over the study area provided TM and ASAR data on 7 July 2008 and 11 July 2008, respectively. ASAR (Advanced Synthetic Aperture Radar) is a synthetic aperture radar carried by the ENVISAT-1 satellite and operates in the C-band (central wavelength $5.63 \mathrm{~cm}$ ), with multi-polarization, seven observation angles and five operating modes. In this study, we chose to use the ASAR data (product code ASAR_APP_1P), and the operating mode was Alternation Polarization corresponding to two kinds of polarization (VV and $\mathrm{VH}$ ) and high space resolution $(12.5 \times 12.5 \mathrm{~m}$ per pixel $)$.

\section{Results}

Two polarizations of ASAR (VV, VH) and all the 7 bands of TM are taken as the input of the classification experiment, and the multisource images are resample to $30 \mathrm{~m} * 30 \mathrm{~m}$ and geometrically corrected. The study area is divided to 10 classes, which are bare farmland, corn, other corps, saline, desert, building, sand, water, mountain, woodland. When the training and validation samples are selected, using the method presented in Section 2.2.2, we can obtain the classification result which is shown in Figure.4

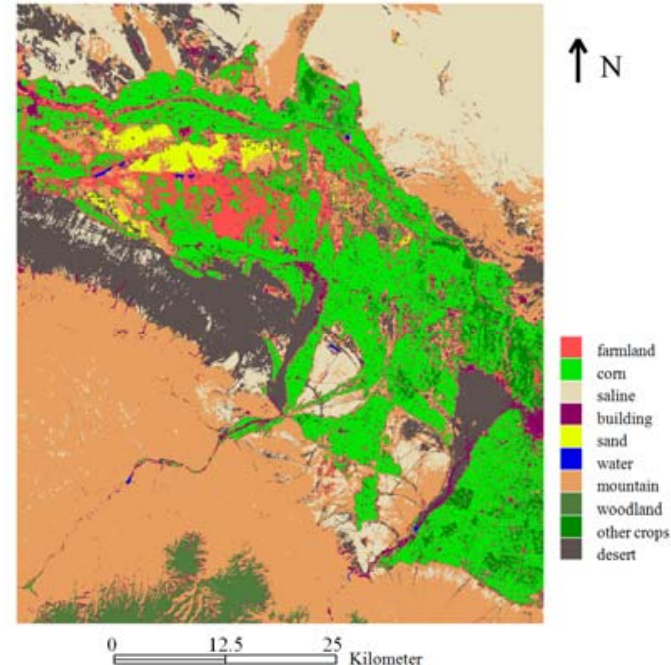

Figure. 4 The Classification map of study area

In Fig. 4, the basic distribution of oasis is consistent with feature of the dual ecological environment of western semiarid regions: 'water always accompanied with oasis, desert accompanied with drought.

We counted the classification of images and found that there were total $1,008,575$ corn pixels, its planting area was $1,008,575 \times 900=907,717,500$ square meters, about $1,361,576$ acres, nearly $18.16 \%$ of the entire study area. There were 153, 922 pixels for other crops, with an planting area $153,922 \times 900=138,529,800$ square meters, about 207,795 acres, nearly $2.77 \%$ of the entire study area. To verify the normalization formula (Eq. (8) and Eq. (12)) and the necessity of coupling optical radar data for classification, we respectively compared the output of the classification of ASAR and TM, using Bayesian network classifier, with the classification only by TM. Table 3 presents the statistical errors among the two methods.

As shown in the Table 2 and Table 3, the accuracy of classification with single TM is low, and reaches a classification precision of $89.63 \%$. When the dual polarization of ASAR data is jointed, the precision increases to $93.72 \%$. The extraction accuracy of corn and other crops planted area by proposed method are $98.47 \%$ and $78.25 \%$, with an improvement of estimation accuracy of about $3.28 \%$ and $4.18 \%$ relative to single $\mathrm{TM}$ classification. The reasons may be that the ASAR 
information can increase the surface characteristics and make them easy to distinguish. For example, corn, other corps, garden and woodland are similar in spectrum, we can identify them by their various structural features caught by ASAR signals and finally obtain a better accuracy. Therefore, the above illustrate that the normalization and integrating optical and SAR data for classification are efficient and reliable

Table 2. Classification by single TM

\begin{tabular}{ccccc}
\hline classes & corn & $\begin{array}{c}\text { other } \\
\text { corps }\end{array}$ & mountain & others \\
\hline corn & 1983 & 308 & 89 & 131 \\
other corps & 146 & 730 & 83 & 84 \\
mountain & 61 & 147 & 469 & 98 \\
others & 36 & 24 & 18 & 12327 \\
sum & 2226 & 1209 & 659 & 12640 \\
\hline
\end{tabular}

a) Total precision $89.63 \%$; Kappa coefficient $87.9 \%$

Table 3. Classification by combining TM and ASAR

\begin{tabular}{ccccc}
\hline classes & corn & $\begin{array}{c}\text { other } \\
\text { corps }\end{array}$ & mountain & others \\
\hline corn & 2192 & 247 & 47 & 55 \\
other corps & 34 & 946 & 9 & 16 \\
mountain & 0 & 16 & 597 & 51 \\
others & 0 & 0 & 6 & 12518 \\
sum & 2226 & 1209 & 659 & 12640 \\
\hline
\end{tabular}

a) Total precision $93.72 \%$, Kappa coefficient $91.07 \%$.

\section{Conclusion}

1) A new classification model for estimation of crop planting area by active and passive remote sensing data is developed in this paper. In the model, the ASAR backscattering coefficient is normalized by the incident angle, then a classifier based on the Bayesian theory is built up, ASAR and TM data are taken as the input of the classifier and the classification experiment is carried out, the validation by field measurements shows that:

2) Compared with the Classification accuracy using single TM, the classification precision of integrating active and passive remote sensing data increases $4.09 \%$, it shows comparing ASAR and TM data can present more information of the objects and increase the classification precision.

3) The extraction accuracy of corn and other crops planted area by proposed method are $98.47 \%$ and $78.25 \%$, with an improvement of estimation accuracy of about $3.28 \%$ and $4.18 \%$ relative to single $\mathrm{TM}$ classification. The results shows the huge potential of crop acreage extracting by active and passive remote sensing data

\section{References}

[1] Yang B. J., 2005. Monitoring agricultural condition by remote sensing. Beijing: Chinese Agriculture Press, pp. 5 - 6.

[2] Sun J. L., 1996. Crop monitoring and the yield estimating by remote sensing in China. Beijing: Chinese Science and Techno logy Press, pp. 12-16

[3] Chen S. p., 1990. The application of remote sensing in agriculture. In: Chen Shupeng. Probe of geography (Volume 3). Application of Remote Sensing. Beijing: Science Press, pp. 20-32

[4] Wu B. F., 2000. Operational Remote Sensing Methods for Agricultural Statistics. ACTA GEOG RAPHICA SINICA, 55(1), pp. 25-35

[5] Donald R. B., Hall F. G., 1980. Global Crop Forecasting. Science, 208, pp. 670-679.

[6] Csornai G., 1999. Operational crop monitoring by remote sensing in Hungary. Nieuwenhuis, Vaughan \&Molenaar Press, pp.20-30

[7] Chen S. B., 1993. Wheat, maize and rice yield estimation techniques Study Collection. Beijing: Science Press,

[8] Fang H. L., 1998A discussion on two strategies applied to estimate rice planting area of an administrative division using remote sensing technique. ACTA GEOG RAPHICA SINICA, 53 (1), pp. 58 - 65.

[9] Wang C. Z., Qi J. G., Susan M., 2004. Soil moisture estimation in a semiarid rangeland using ERS-2 and TM imagery. remote sensing of environment, 90, pp.178-289

[10] Wu T. D., Chen K .S., 2004 A reappraisal of the validity of the IEM model for backscattering from rough surface. IEEE Transactions on Geoscience and Remote Sensing, 42, pp. 743-753

[11] Fung A. K., 1992Backscattering from a randomly rough dielectric surface. IEEE Transactions on Geoscience and Remote Sensing, 30., pp. $56-369$

[12] Li Q., Shi J. C., 2002. A generalized power law spectrum and its applications to the backscattering of soil surfaces based on the integral equation model. IEEE Transactions on Geoscience and Remote Sensing, 40, pp. 271-280

[13] Ulaby F. T., Moore R. K., Fung A. K., 1982. Microwave remote sensing. Volume II :Radar Remote Sensing and surface Scattering and Emission Theory. Addison-Wesley Publishing Company Press, 123-126 